\title{
Object Recognition with and without Objects
}

\author{
Zhuotun Zhu, Lingxi Xie, Alan Yuille \\ Johns Hopkins University, Baltimore, MD, USA \\ \{zhuotun, 198808xc, alan.l.yuille\}@gmail.com
}

\begin{abstract}
While recent deep neural networks have achieved a promising performance on object recognition, they rely implicitly on the visual contents of the whole image. In this paper, we train deep neural networks on the foreground (object) and background (context) regions of images respectively. Considering human recognition in the same situations, networks trained on the pure background without objects achieves highly reasonable recognition performance that beats humans by a large margin if only given context. However, humans still outperform networks with pure object available, which indicates networks and human beings have different mechanisms in understanding an image. Furthermore, we straightforwardly combine multiple trained networks to explore different visual cues learned by different networks. Experiments show that useful visual hints can be explicitly learned separately and then combined to achieve higher performance, which verifies the advantages of the proposed framework.
\end{abstract}

\section{Introduction}

Object recognition is a long-lasting battle in computer vision, which aims to categorize an image according to the visual contents. In recent years, we have witnessed an evolution in this research field. Thanks to the availability of large-scale image datasets [Deng et al., 2009] and powerful computational resources, it becomes possible to train a very deep convolutional neural network (CNN) [Krizhevsky et al., 2012], which is much more efficient beyond the conventional Bagof-Visual-Words (BoVW) model [Csurka et al., 2004].

It is known that an image contains both foreground and background visual contents. However, most object recognition algorithms focus on recognizing the visual patterns only on the foreground region [Zeiler and Fergus, 2014]. Although it has been proven that background (context) information also helps recognition [Simonyan and Zisserman, 2014], it still remains unclear if a deep network can be trained individually to learn visual information only from the background region. In addition, we are interested in exploring different visual patterns by training neural networks on foreground and back-

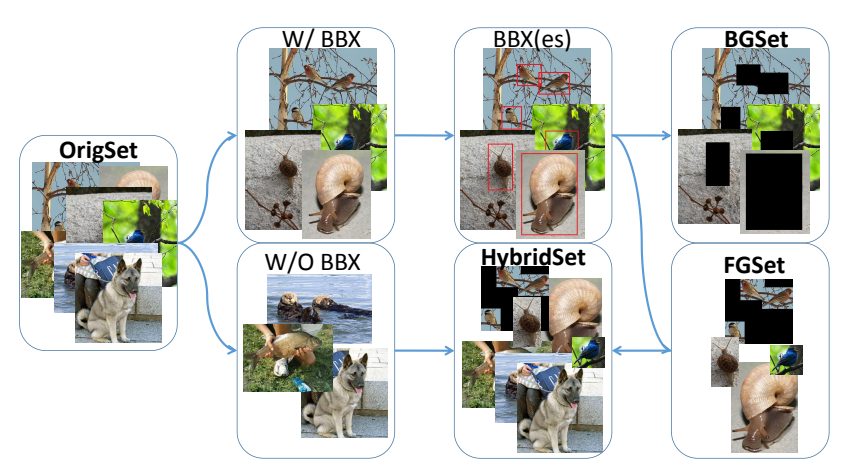

Figure 1: Procedures of dataset generation. First, we denote the original set as the OrigSet, divided into two sets, one with the ground-truth bounding box (W/ BBX) and the other one without (W/O BBX). Then the set with labelled bounding box(es) are further processed by setting regions inside all ground-truth to be 0 's to compose the BGSet while cropping the regions out to produce the FGSet. In the end, add the images without bounding boxes with FGSet to construct the HybridSet. Please note that some images of the FGSet have regions to be black (0's) since these images are labelled with multiple objects belonging to the same class, which are cropped according to the smallest rectangle frame that includes all object bounding boxes in order to keep as less background information as possible on FGSet. Best viewed in color.

ground separately for object recognition, which is less studied before.

In this work, we investigate the above problems by explicitly training multiple networks for object recognition. We first construct datasets from ILSVRC2012 [Russakovsky et al., 2015], i.e., one foreground set and one background set, by taking advantage of the ground-truth bounding box(es) provided in both training and testing cases. After dataset construction, we train deep networks individually to learn foreground (object) and background (context) information, respectively. We find that, even only trained on pure background contexts, the deep network can still converge and makes reasonable prediction (14.4\% top-1 and nearly $\mathbf{3 0} \%$ top- 5 classification accuracy on the background validation set). To make a comparison, we are further interested in the human recognition performance on the constructed datasets. Deep neural networks outperform non-expert humans in finegrained recognition, and humans sometimes make errors be- 
cause they cannot memorize all categories of datasets [Russakovsky et al., 2015]. In this case, to more reasonably compare the recognition ability of humans and deep networks, we follow [Huh et al., 2016] to merge all the 1,000 fine-grained categories of the original ILSVRC2012, resulting in a 127class recognition problem meanwhile keeping the number of training/testing images unchanged. We find that human beings tend to pay more attention to the object while networks put more emphasis on context than humans for classification. By visualizing the patterns captured by the background net, we find that some visual patterns are not available in the foreground net. Therefore, we apply networks on the foreground and background regions respectively via the given groundtruth bounding box(es) or extracting object proposals without available ones. We find that the linear combination of multiple neural networks can give higher performance.

To summarize, our main contributions are three folds: 1) We demonstrate that learning foreground and background visual contents separately is beneficial for object recognition. Training a network based on pure background although being wired and challenging, is technically feasible and captures highly useful visual information. 2) We conduct $\boldsymbol{h u}$ man recognition experiments on either pure background or foreground regions to find that human beings outperform networks on pure foreground while are beaten by networks on pure background, which implies the different mechanisms of understanding an image between networks and humans. 3) We straightforwardly combine multiple neural networks to explore the effectiveness of different learned visual clues under two conditions with and without ground-truth bounding box(es), which gives promising improvement over the baseline deep neural networks.

\section{Related Work}

Object recognition is fundamental in computer vision field, which is aimed to understand the semantic meaning among an image via analyzing its visual contents. Recently, researchers have extended the traditional cases [Lazebnik et al., 2006] to fine-grained [Wah et al., 2011] [Nilsback and Zisserman, 2008] [Lin et al., 2015], and large-scale [Xiao et al., 2010] [Griffin et al., 2007] tasks. Before the exploding development of deep learning, the dominant BoVW model [Csurka et al., 2004] represents every single image with a high-dimensional vector. It is typically composed of three consecutive steps, i.e., descriptor extraction [Lowe, 2004] [Dalal and Triggs, 2005], feature encoding [Wang et al., 2010] [Perronnin et al., 2010] and feature summarization [Lazebnik et al., 2006].

The milestone Convolutional Neural Network (CNN) is treated as a hierarchical model for large-scale visual recognition. In past years, neural networks have already been proved to be effective for simple recognition tasks [LeCun et al., 1990]. More recently, the availability of large-scale training data (e.g., ImageNet [Deng et al., 2009]) and powerful computation source like GPUs make it practical to train deep neural networks [Krizhevsky et al., 2012] [Zhu et al., 2016] [Fang et al., 2015] [He et al., 2016b] which significantly outperform the conventional models. Even deep fea- tures have been proved to be very successful on vision tasks like object discovery [Wang et al., 2015b], object recognition [Xie et al., 2017], etc. A CNN is composed of numerous stacked layers, in which responses from the previous layer are then convoluted and activated by a differentiable function, followed by a non-linear transformation [Nair and Hinton, 2010] to avoid over-fitting. Recently, several efficient methods were proposed to help CNNs converge faster and prevent over-fitting [Krizhevsky et al., 2012]. It is believed that deeper networks produce better recognition results [Szegedy et al., 2015][Simonyan and Zisserman, 2014], but also requires engineering tricks to be trained very well [Ioffe and Szegedy, 2015] [He et al., 2016a].

Very few techniques on background modeling [Bewley and Upcroft, 2017] have been developed for object recognition, despite the huge success of deep learning methods on various vision tasks. [Shelhamer et al., 2016] proposed the fully convolutional networks (FCN) for semantic segmentation, which are further trained on foreground and background defined by shape masks. They find it is not vital to learn a specifically designed background model. For face matching, [Sanderson and Lovell, 2009] developed methods only on the cropped out faces to alleviate the possible correlations between faces and their backgrounds. [Han et al., 2015] modeled the background in order to detect the salient objects from the background. [Doersch et al., 2014] showed using the object patch to predict its context as supervisory information can help discover object clusters, which is consistent with our motivation to utilize the pure context for visual recognition. To our best knowledge, we are the first to explicitly learn both the foreground and background models and then combine them together to be beneficial for the object recognition.

Recently, researchers pay more attention to human experiments on objects recognition. Zhou et al. [Zhou et al., 2015] invited Amazon Mechanical Turk (AMT) to identify the concept for segmented images with objects. They found that the CNN trained for scene classification automatically discovers meaningful object patches. While in our experiments, we are particularly interested in the different emphasis between human beings and networks for recognition task.

Last but not the least, visualization of CNN activations is an effective method to understand the mechanism of CNNs. In [Zeiler and Fergus, 2014], a de-convolutional operation was proposed to capture visual patterns on different layers of a trained network. [Simonyan and Zisserman, 2014] and [Cao et al., 2015] show that different sets of neurons are activated when a network is used for detecting different visual patterns. In this work, we will use a much simpler way of visualization which is inspired by [Wang et al., 2015a].

\section{Training Networks}

Our goal is to explore the possibility and effectiveness of training networks on foreground and background regions, respectively. Here, foreground and background regions are defined by the annotated ground-truth bounding box(es) of each image. All the experiments are done on the datasets composed from the ILSVRC2012. 
Proceedings of the Twenty-Sixth International Joint Conference on Artificial Intelligence (IJCAI-17)

\begin{tabular}{lcrcc}
\hline Dataset & Image Description & \# Training Image & \# Testing Image & Testing Accuracy \\
\hline OrigSet & Original Image & $1,281,167$ & 50,000 & $58.19 \%, 80.96 \%$ \\
FGSet & Foreground Image & 544,539 & 50,000 & $60.82 \%, 83.43 \%$ \\
BGSet & Background Image & 289,031 & 50,000 & $14.41 \%, 29.62 \%$ \\
HybridSet & Original Image or Foreground Image & $1,281,167$ & 50,000 & $61.29 \%, 83.85 \%$ \\
\hline
\end{tabular}

Table 1: The configuration of different image datasets originated from the ILSVRC2012. The lass column denotes the testing performance of trained AlexNet in terms of top- 1 and top-5 classification accuracy on corresponding datasets, e.g., the BGNet gives $14.41 \%$ top- 1 and $29.62 \%$ top- 5 accuracy on the testing images of BGSet.

\subsection{Data Preparation}

The ILSVRC2012 dataset [Russakovsky et al., 2015] contains about $1.3 \mathrm{M}$ training and $50 \mathrm{~K}$ validation images. Throughout this paper, we refer to the original dataset as OrigSet and the validation images are regarded as our testing set. Among OrigSet, 544,539 training images and all 50,000 testing images are labeled with at least one groundtruth bounding box. For each image, there is only one type of object annotated according to its ground-truth class label.

We construct three variants of training sets and two variants of testing sets from OrigSet by details below. An illustrative example of data construction is shown in Figure 1. The configuration of different image datasets are summarized in Table 1.

- The foreground dataset (FGSet) is composed of all images with at least one available ground-truth bounding box. For each image, we first compute the smallest rectangle frame which includes all object bounding boxes, then based on which the image inside the frame is cropped to be used as the training/testing data. Note that if an image has multiple object bounding boxes belonging to the same class, we set all the background regions inside the frame to be 0's to keep as little context as possible on FGSet. There are totally 544,539 training images and 50,000 testing images on FGSet. Since the annotation is on the bounding box level, images of the FGSet may contain some background information.

- The construction of the background dataset (BGSet) consists of two stages. First, for each image with at least one ground-truth bounding box available, regions inside every ground-truth bounding box are set to 0 's. Chances are that almost all the pixels of one image are set to $0 \mathrm{~s}$ if its object consists of nearly 100 percent of its whole region. Therefore during training, we discard those samples with less than $50 \%$ background pixels preserved, i.e., the foreground frame is larger than half of the entire image, so that we can maximally prevent using those less meaningful background contents (see Figure 1). However in testing, we keep all the processed images, in the end, 289,031 training images and 50,000 testing images are preserved.

- To increase the amount of training data for foreground classification, we also construct a hybrid dataset, abbreviated as the HybridSet. The HybridSet is composed of all images of the original training set. If at least one ground-truth bounding box is available, we pre-process this image as described on FGSet, otherwise, we simply keep this image without doing anything. As bounding box annotation is available in each testing case, the HybridSet and the FGSet contain the same testing data. Training with the HybridSet can be understood as a semi-supervised learning process.

\subsection{Training and Testing}

We trained the milestone AlexNet [Krizhevsky et al., 2012] using the CAFFE library [Jia et al., 2014] on different training sets as mentioned in the $\mathrm{Sec} 3.1$.

The base learning rate is set to 0.01 , and reduced by $1 / 10$ for every 100,000 iterations. The moment is set to be 0.9 and the weight decay parameter is 0.0005 . A total number of 450,000 iterations is conducted, which corresponds to around 90 training epochs on the original dataset. Note that both FGSet and BGSet contain less number of images than that of OrigSet and HybridSet, which leads to a larger number of training epochs, given the same training iterations. In these cases, we adjust the dropout ratio as 0.7 to avoid the overfitting issue. We refer to the network trained on the OrigSet as the OrigNet, and similar abbreviated names also apply to other cases, i.e., the FGNet, BGNet and HybridNet.

During testing, we report the results by using the common data augmentation of averaging 10 patches from the 5 crops and 5 flips. After all forward passes are done, the average output on the final $(f c-8)$ layer is used for prediction. We adopt the MatConvNet [Vedaldi and Lenc, 2015] platform for performance evaluation.

\section{Experiments}

The testing accuracy of AlexNet trained on corresponding dataset are given in the last column of Table 1. We can find that the BGNet produces reasonable classification results: $14.41 \%$ top- 1 and $29.62 \%$ top- 5 accuracy (while the random guess gets $0.1 \%$ and $0.5 \%$, respectively), which is a bit surprising considering it makes classification decisions only on background contents without any foreground objects given. This demonstrates that deep neural networks are capable of learning pure contexts to infer objects even being fully occluded. Not surprisingly, the HybridNet gives better performance than the FGNet due to more training data available.

\subsection{Human Recognition}

As stated before, to alleviate the possibility of wrongly classifying images for humans beings due to high volume of classes up to 1,000 on the original ILSVRC2012, we follow [Huh et al., 2016] by merging all the fine-grained categories, resulting 


\begin{tabular}{lcc}
\hline Dataset & AlexNet & Human \\
\hline OrigSet & $58.19 \%, 80.96 \%$ &,$- 94.90 \%^{\star}$ \\
BGSet & $14.41 \%, 29.62 \%$ &,-- \\
\hline OrigSet-127 & $73.16 \%, 93.28 \%$ &,-- \\
FGSet-127 & $75.32 \%, 93.87 \%$ & $81.25 \%, 95.83 \%$ \\
BGSet-127 & $41.65 \%, 73.79 \%$ & $18.36 \%, 39.84 \%$ \\
\hline
\end{tabular}

Table 2: Classification accuracy (in terms of top-1, top-5) on five sets by deep neural networks and human, respectively.

\begin{tabular}{lccc}
\hline Network & OrigSet & FGSet & BGSet \\
\hline OrigNet & $\mathbf{5 8 . 1 9} \%, \mathbf{8 0 . 9 6} \%$ & $50.73 \%, 74.11 \%$ & $3.83 \%, 9.11 \%$ \\
FGNet & $33.42 \%, 53.72 \%$ & $60.82 \%, 83.43 \%$ & $1.44 \%, 4.53 \%$ \\
BGNet & $4.26 \%, 10.73 \%$ & $1.69 \%, 5.34 \%$ & $\mathbf{1 4 . 4 1} \%, \mathbf{2 9 . 6 2} \%$ \\
HybridNet $52.89 \%, 76.61 \%$ & $\mathbf{6 1 . 2 9} \%, \mathbf{8 3 . 8 5} \%$ & $3.48 \%, 9.05 \%$ \\
\hline
\end{tabular}

Table 3: Cross evaluation accuracy (in terms of top-1, top-5) on four networks and three testing sets. Note that the testing set of HybridSet is identical to that of FGSet.

in a 127-class recognition problem meanwhile keeping the number of training/testing images unchanged. To distinguish the merged 127-class datasets with the previous datasets, we refer to them as the OrigSet-127, FSet-127 and BGSet-127, respectively. Then we invite volunteers who are familiar with the merged 127 classes to perform the recognition task on BGSet-127 and FSet-127. Humans are given 256 images covering all 127 classes and one image takes around two minutes to make the top- 5 decisions. We do not evaluate humans on OrigSet-127 since we believe humans can perform well on this set like on OrigSet. Human performance on OrigSet (labeled by ${ }^{\star}$ ) is reported by [Russakovsky et al., 2015].

Table 2 gives the testing recognition performance of human beings and trained AlexNet on different datasets. It is well noted that humans are good at recognizing natural images [Russakovsky et al., 2015], e.g., on OrigSet, human labelers achieve much higher performance than AlexNet. We can find the human beings also surpass networks on the foreground (object-level) recognition by $5.93 \%$ and $1.96 \%$ in terms of top- 1 and top- 5 accuracy. Surprisingly, AlexNet beats human labelers to a large margin on the background dataset BGSet-127 considering the $127 \%$ and $85 \%$ relative improvements from $18.36 \%$ to $41.65 \%$ and $39.84 \%$ to $73.79 \%$ for top- 1 and top- 5 accuracy, respectively. In this case, the networks are capable of exploring background hints for recognition much better than human beings. On the contrary, humans classify images mainly based on the visual contents of the foreground objects.

\subsection{Cross Evaluation}

To study the difference in visual patterns learned by different networks, we perform the cross evaluation, i.e., applying each trained network to different testing sets. Results are summarized in Table 3.

We find that the transferring ability of each network is limited, since a model cannot obtain satisfying performance in the scenario of different distributions between training and testing data. For example, using FGNet to predict OrigSet leads to $27.40 \%$ absolute drop ( $45.05 \%$ relative) in top- 1 accuracy, meanwhile using OrigNet to predict FGSet leads to $7.46 \%$ drop ( $12.82 \%$ relative) in top- 1 accuracy. We conjecture that FGNet may store very little information on contexts, thus confused by the background context of OrigSet. On the other side, OrigNet has the ability of recognizing contexts but is wasted for the task on FGSet.
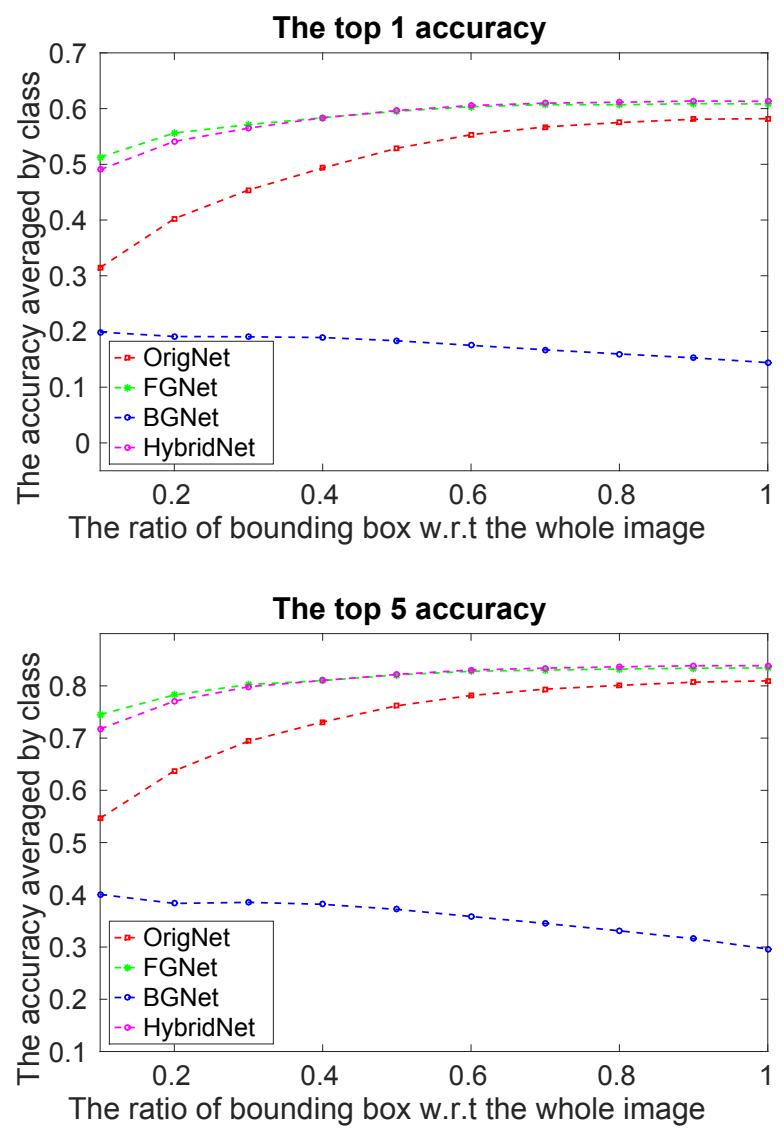

Figure 2: Classification accuracy with respect to the foreground ratio on testing images. The number at, say, 0.3 , represents the testing accuracy on the set of all images with foreground ratio no greater than $30 \%$. Best viewed in color.

\subsection{Diagnosis}

We conduct diagnostic experiments to study the property of different networks to fully understand the networks behaviors. Specifically, we report the classification accuracy of different networks with respect to keeping different foreground ratios of the testing image.

We split each testing dataset into 10 subsets, each of which contains all images with the foreground ratio no greater than a fixed value. Results are shown in Figure 2. BGNet gets higher classification accuracy on the images with a relatively smaller foreground ratio, while other three networks prefer a large object ratio since the foreground information is primarily learned for recognition in these cases. Furthermore when the foreground ratio goes larger, e.g., greater than $80 \%$, 
Proceedings of the Twenty-Sixth International Joint Conference on Artificial Intelligence (IJCAI-17)

\begin{tabular}{lcc}
\hline Network & Guided & Unguided \\
\hline OrigNet & $58.19 \%, 80.96 \%$ & $58.19 \%, 80.96 \%$ \\
BGNet & $14.41 \%, 29.62 \%$ & $8.30 \%, 20.60 \%$ \\
FGNet & $60.82 \%, 83.43 \%$ & $40.71 \%, 64.12 \%$ \\
HybridNet & $61.29 \%, 83.85 \%$ & $45.58 \%, 70.22 \%$ \\
\hline FGNet+BGNet & $61.75 \%, 83.88 \%$ & $41.83 \%, 65.32 \%$ \\
HybridNet+BGNet & $62.52 \%, 84.53 \%$ & $48.08 \%, 72.69 \%$ \\
HybridNet+OrigNet & $\mathbf{6 5 . 6 3} \%, \mathbf{8 6 . 6 9} \%$ & $\mathbf{6 0 . 3 6} \%, \mathbf{8 2 . 4 7} \%$ \\
\hline
\end{tabular}

Table 4: Classification accuracy (in terms of top-1, top-5) comparison of different network combinations. It's worth noting that we feed the entire image into the OrigNet no matter whether the groundtruth bounding box(es) is given in order to keep the testing phase consistent with the training of OrigNet. Therefore, the reported results of OrigNet are same with each other under both guided and unguided conditions. To integrate the results from several networks, we weighted sum up the responses on the $f c-8$ layer.

the performance gap among OrigNet, FGNet and HybridNet gets smaller.

\subsection{Visualization}

In this part, we visualize the networks to see how different networks learn different visual patterns. We adopt a very straightforward visualization method [Wang et al., 2015a], which takes a trained network and reference images as input.

We visualize the most significant responses of the neurons on the conv-5 layer. The conv-5 layer is composed of 256 filter response maps, each of which has $13 \times 13$ different spatial positions. After all the 50,000 reference images are processed, we obtain $13^{2} \times 50000$ responses for each of the 256 filters. We pick up those neurons with the highest response and trace back to obtain its receptive field on the input image. In this way, we can discover the visual patterns that best describe the concept this filter has learned. For diversity, we only choose at most one patch from a reference image with the highest response score.

Figure 3 shows visualization results using FGNet on FGSet, BGNet on BGSet and OrigNet on OrigSet, respectively. We can observe quite different visual patterns learned by these networks. The visual patterns learned by FGNet are often very specific to some object categories, such as the patch of a dog face (filter 5) or the front side of a shop (filter 11). These visual patterns correspond to some visual attributes, which are vital for recognition. However, each visual concept learned by BGNet tends to appear in many different object categories, for instance, the patch of outdoor scene (filter 8) shared by the jetty, viaduct, space shuttle, etc. These visual patterns are often found in the context, which plays an assistant role in object recognition. As for OrigNet, the learned patterns can be shared specific objects or scene.

To summarize, FGNet and BGNet learn different visual patterns that can be combined to assist visual recognition. In Sec 4.3 we quantitatively demonstrate the effectiveness of these networks via combining these information for better recognition performance.

\section{Combination}

We first show that the recognition accuracy can be significantly boosted using ground-truth bounding box(es) at the testing stage. Next, with the help of the EdgeBox algorithm [Zitnick and Dollar, 2014] to generate accurate object proposals, we improve the recognition performance without the requirement of ground-truth annotations. We name them as guided and unguided combination, respectively.

\subsection{Guided vs. Unguided Combination}

We start with describing guided and unguided manners of the model combination. For simplicity, we adopt the linear combination over different models, i.e., forwarding several networks, and weighted summing up the responses on the $f c-8$ layer.

If the ground-truth bounding box is provided (the guided condition), we use the ground-truth bounding box to divide the testing image into foreground and background regions. Then, we feed the foreground regions into FGNet or HybridNet, and background regions into BGNet, then fuse the neuron responses at the final stage.

Furthermore, we also explore the solution of combining multiple networks in an unguided manner. As we will see in Sec 5.2, a reliable bounding box helps a lot in object recognition. Motivated by which, we use an efficient and effective algorithm, EdgeBox, to generate a lot of potential bounding boxes proposals for each testing image, and then feed the foreground and background regions into neural networks as described before across top proposals.

To begin with, we demonstrate the EdgeBox proposals are good to capture the ground-truth object. After extracting top$k$ proposals with EdgeBox, we count the detected groundtruth if at least one of proposals has the IoU no less than 0.7 with the ground-truth. The cumulative distribution function (CDF) is plotted in Figure 4. Considering efficiency as well as accuracy, we choose the top-100 proposals to feed the foreground and background into trained networks, which give an around $81 \%$ recall. After obtaining 100 outputs for each network, we average responses of $f c-8$ layer for classification.

\subsection{Combination Results and Discussion}

Results of different combinations are summarized in Table 4. Under either guided or unguided settings, combining multiple networks boosts recognition performance, which verifies the statement that different visual patterns from different networks can help with each other for the object recognition.

Take a closer look at the accuracy gain under the unguided condition. The combination of HybridNet + BGNet outperforms HybridNet by $2.50 \%$ and $2.47 \%$ in terms of top- 1 and top- 5 recognition accuracy, which are noticeable gains. As for the FGNet+BGNet, it improves $1.12 \%$ and $1.20 \%$ classification accuracy compared with the FGNet, which are promising. Surprisingly, the combination of $\mathbf{H y -}$ bridNet with OrigNet can still increase from the OrigNet by $2.17 \%$ and $1.51 \%$. We hypothesize that the combination is capable of discovering the objects implicitly by the inference of where the objects are due to the visual patterns of HybridNet are learned from images with object spatial 

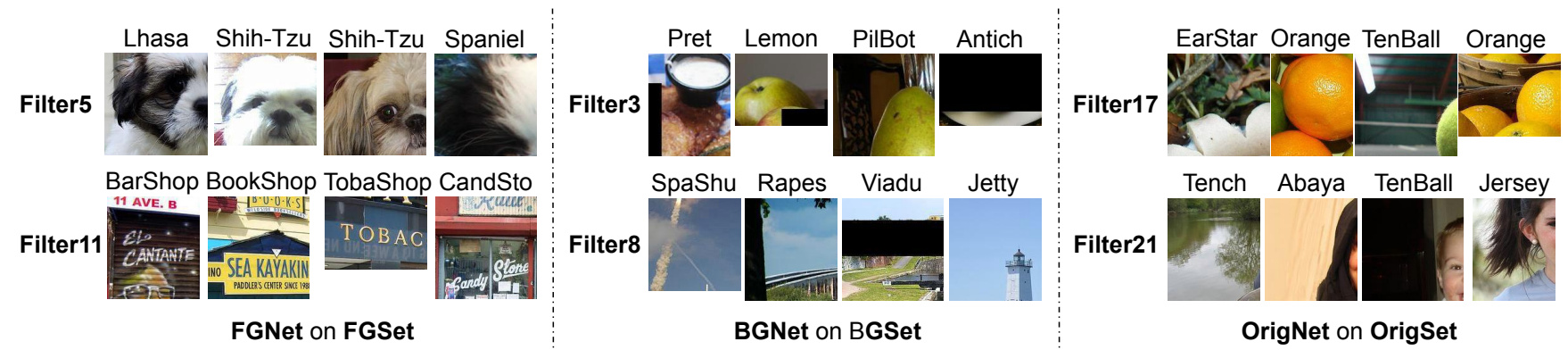

Figure 3: Patch visualization of FGNet on FGSet (left), BGNet on BGSet (middle) and OrigNet on OrigSet (right). Each row corresponds to one filter on the conv-5 layer, and each patch is selected from $13^{2} \times 50000$ ones, with the highest response on that kernel. Best viewed in color.

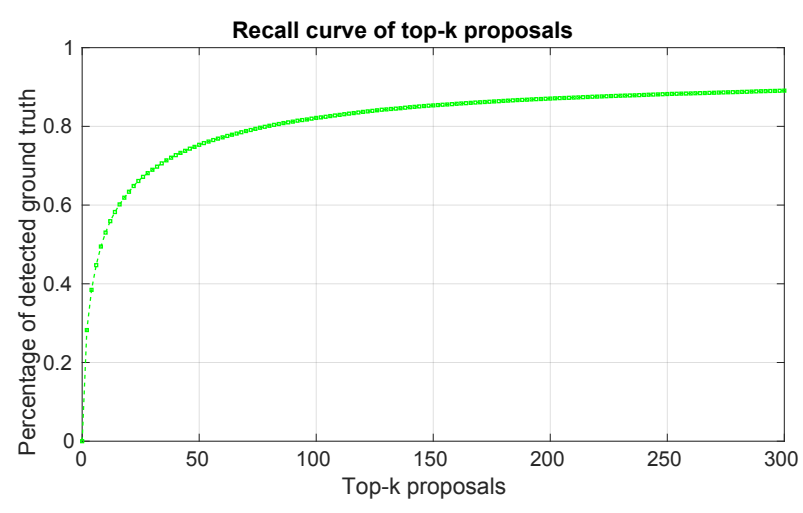

Figure 4: EdgeBox statistics on ILSVRC2012 validation set, which denotes the curriculum distribution function of the detected groundtruth with respect to the top- $k$ proposals. Here, we set the Intersection over Union (IoU) threshold to be 0.7 for EdgeBox algorithm.

information. One may conjecture that the performance improvement may come from the ensemble effect, which is not necessarily true considering: 1) object proposals are not accurate enough; 2) data augmentation (5 crops and 5 flips) is already done for the OrigNet, therefore the improvement is complementary to data augmentation. Moreover, we quantitatively verify that the improvements are not from simple data augmentation by giving the results of OrigNet averaged by 100 densely sampled patches (50 crops and corresponding 50 flips, $227 \times 227 \times 3$, referred to as OrigNet100) instead of the default ( 5 crops and 5 flips) setting. The top- 1 and top- 5 accuracy of OrigNet100 are $58.08 \%$ and $81.05 \%$, which are very similar to original $58.19 \%$ and $80.96 \%$. This suggests that the effect of data augmentation by 100 patches is negligible. By contrast, HybridNet+OrigNet100 reports $60.80 \%$ and $82.59 \%$, significantly higher than OrigNet100 alone, which reveals that HybridNet brings in some benefits that are not achieved via data augmentation. These improvements are super promising considering that the networks don't know where the accurate objects are under the unguided condition. Notice that the results under unguided condition cannot surpass those under guided condition, arguably because the top- 100 proposals not good enough to capture the accurate ground-truth given that the BGNet cannot give high confidence on the predictions.

For the guided way of testing, by providing accurate separation of foreground from background, works better than the unguided way by a large margin, which makes sense. And the improvements can consistently be found after combinations with the BGNet. It is well worth noting that the combination of HybridNet with OrigGNet improves the baseline of OrigGNet to a significant margin by $7.44 \%$ and $5.73 \%$. The huge gains are reasonable because of networks' ability to infer object locations trained on accurate bounding box(es).

\section{Conclusions and Future Work}

In this work, we first demonstrate the surprising finding that neural networks can predict object categories quite well even when the object is not present. This motivates us to study the human recognition performance on foreground with objects and background without objects. We show on the 127-classes ILSVRC2012 that human beings beat neural networks for foreground object recognition, while perform much worse to predict the object category only on the background without objects. Then explicitly combining the visual patterns learned from different networks can help each other for the recognition task. We claim that more emphasis should be placed on the role of contexts for object detection and recognition.

In the future, we will investigate an end-to-end training approach for explicitly separating and then combining the foreground and background information, which explores the visual contents to the full extent. For instance, inspired by some joint learning strategy such as Faster R-CNN [Ren et $a l ., 2015]$, we can design a structure which predicts the object proposals in the intermediate stage, then learns the foreground and background regions derived from the proposals separately by two sub-networks and then takes foreground and background features into further consideration.

\section{Acknowledgments}

This work is supported by the Intelligence Advanced Research Projects Activity (IARPA) via Department of Interior/Interior Business Center (DoI/IBC) contract number D16PC00007. We greatly thank the anonymous reviewers and JHU CCVL members who have given valuable and constructive suggestions which make this work better. 


\section{References}

[Bewley and Upcroft, 2017] A. Bewley and B. Upcroft. Background appearance modeling with applications to visual object detection in an open-pit mine. JFR, 2017.

[Cao et al., 2015] C. Cao, X. Liu, Y. Yang, Y. Yu, J. Wang, Z. Wang, Y. Huang, L. Wang, C. Huang, and W. Xu. Look and Think Twice: Capturing Top-Down Visual Attention with Feedback Convolutional Neural Networks. ICCV, 2015.

[Csurka et al., 2004] G. Csurka, C. Dance, L. Fan, J. Willamowski, and C. Bray. Visual Categorization with Bags of Keypoints. Workshop on ECCV, 2004.

[Dalal and Triggs, 2005] N. Dalal and B. Triggs. Histograms of Oriented Gradients for Human Detection. CVPR, 2005.

[Deng et al., 2009] J. Deng, W. Dong, R. Socher, L.J. Li, K. Li, and L. Fei-Fei. ImageNet: A Large-Scale Hierarchical Image Database. CVPR, 2009.

[Doersch et al., 2014] C. Doersch, A. Gupta, and A.A. Efros. Context as supervisory signal: Discovering objects with predictable context. ECCV, 2014.

[Fang et al., 2015] Y. Fang, J. Xie, G. Dai, M. Wang, F. Zhu, T. Xu, and E. Wong. 3d deep shape descriptor. In CVPR, 2015.

[Griffin et al., 2007] G. Griffin, A. Holub, and P. Perona. Caltech256 Object Category Dataset. Technical Report: CNS-TR-2007001, Caltech, 2007.

[Han et al., 2015] J. Han, D. Zhang, X. Hu, L. Guo, J. Ren, and F. Wu. Background prior-based salient object detection via deep reconstruction residual. TCSVT, 2015.

[He et al., 2016a] K. He, X. Zhang, S. Ren, and J. Sun. Deep Residual Learning for Image Recognition. CVPR, 2016.

[He et al., 2016b] K. He, X. Zhang, S. Ren, and J. Sun. Identity mappings in deep residual networks. In ECCV, 2016.

[Huh et al., 2016] M. Huh, P. Agrawal, and A.A. Efros. What Makes ImageNet Good for Transfer Learning? arXiv: 1608.08614, 2016.

[Ioffe and Szegedy, 2015] S. Ioffe and C. Szegedy. Batch Normalization: Accelerating Deep Network Training by Reducing Internal Covariate Shift. ICML, 2015.

[Jia et al., 2014] Y. Jia, E. Shelhamer, J. Donahue, S. Karayev, J. Long, R. Girshick, S. Guadarrama, and T. Darrell. CAFFE: Convolutional Architecture for Fast Feature Embedding. ACM $M M, 2014$.

[Krizhevsky et al., 2012] A. Krizhevsky, I. Sutskever, and G.E. Hinton. ImageNet Classification with Deep Convolutional Neural Networks. NIPS, 2012.

[Lazebnik et al., 2006] S. Lazebnik, C. Schmid, and J. Ponce. Beyond Bags of Features: Spatial Pyramid Matching for Recognizing Natural Scene Categories. CVPR, 2006.

[LeCun et al., 1990] Y. LeCun, J.S. Denker, D. Henderson, R.E. Howard, W. Hubbard, and L.D. Jackel. Handwritten Digit Recognition with a Back-Propagation Network. NIPS, 1990.

[Lin et al., 2015] T. Lin, A. RoyChowdhury, and S. Maji. Bilinear $\mathrm{cnn}$ models for fine-grained visual recognition. ICCV, 2015.

[Lowe, 2004] D.G. Lowe. Distinctive Image Features from ScaleInvariant Keypoints. IJCV, 2004.

[Nair and Hinton, 2010] V. Nair and G.E. Hinton. Rectified linear units improve restricted boltzmann machines. ICML, 2010.
[Nilsback and Zisserman, 2008] M.E. Nilsback and A. Zisserman. Automated Flower Classification over a Large Number of Classes. ICVGIP, 2008.

[Perronnin et al., 2010] F. Perronnin, J. Sanchez, and T. Mensink. Improving the Fisher Kernel for Large-scale Image Classification. $E C C V, 2010$.

[Ren et al., 2015] S. Ren, K. He, R. Girshick, and J. Sun. Faster RCNN: Towards Real-time Object Detection with Region Proposal Networks. NIPS, 2015.

[Russakovsky et al., 2015] O. Russakovsky, J. Deng, H. Su, J. Krause, S. Satheesh, S. Ma, Z. Huang, A. Karpathy, A. Khosla, M. Bernstein, A.C. Berg, and L. Fei-Fei. ImageNet Large Scale Visual Recognition Challenge. IJCV, pages 1-42, 2015.

[Sanderson and Lovell, 2009] C. Sanderson and B.C. Lovell. Multi-region probabilistic histograms for robust and scalable identity inference. $I C B, 2009$.

[Shelhamer et al., 2016] E. Shelhamer, J. Long, and T. Darrell. Fully convolutional networks for semantic segmentation. TPAMI, 2016.

[Simonyan and Zisserman, 2014] K. Simonyan and A. Zisserman. Very Deep Convolutional Networks for Large-Scale Image Recognition. ICLR, 2014.

[Szegedy et al., 2015] C. Szegedy, W. Liu, Y. Jia, P. Sermanet, S. Reed, D. Anguelov, D. Erhan, V. Vanhoucke, and A. Rabinovich. Going Deeper with Convolutions. CVPR, 2015.

[Vedaldi and Lenc, 2015] A. Vedaldi and K. Lenc. MatConvNet Convolutional Neural Networks for MATLAB. In ACM MM, 2015.

[Wah et al., 2011] C. Wah, S. Branson, P. Welinder, P. Perona, and S. Belongie. The Caltech-UCSD Birds-200-2011 Dataset. Technical Report: CNS-TR-2011-001, Caltech, 2011.

[Wang et al., 2010] J. Wang, J. Yang, K. Yu, F. Lv, T. Huang, and Y. Gong. Locality-Constrained Linear Coding for Image Classification. CVPR, 2010.

[Wang et al., 2015a] J. Wang, Z. Zhang, V. Premachandran, and A. Yuille. Discovering Internal Representations from ObjectCNNs Using Population Encoding. arXiv: 1511.06855, 2015.

[Wang et al., 2015b] X. Wang, Z. Zhu, C. Yao, and X. Bai. Relaxed multiple-instance svm with application to object discovery. ICCV, 2015

[Xiao et al., 2010] J. Xiao, J. Hays, K.A. Ehinger, A. Oliva, and A. Torralba. SUN Database: Large-Scale Scene Recognition from Abbey to Zoo. CVPR, 2010.

[Xie et al., 2017] L. Xie, J. Wang, W. Lin, B. Zhang, and Q. Tian. Towards reversal-invariant image representation. IJCV, 2017.

[Zeiler and Fergus, 2014] M.D. Zeiler and R. Fergus. Visualizing and Understanding Convolutional Networks. ECCV, 2014.

[Zhou et al., 2015] B. Zhou, A. Khosla, A. Lapedriza, A. Oliva, and A. Torralba. Object Detectors Emerge in Deep Scene CNNs. ICLR, 2015

[Zhu et al., 2016] Z. Zhu, X. Wang, S. Bai, C. Yao, and X. Bai. Deep learning representation using autoencoder for $3 \mathrm{~d}$ shape retrieval. Neurocomputing, 2016.

[Zitnick and Dollar, 2014] C.L. Zitnick and P. Dollar. Edge Boxes: Locating Object Proposals from Edges. ECCV, 2014. 